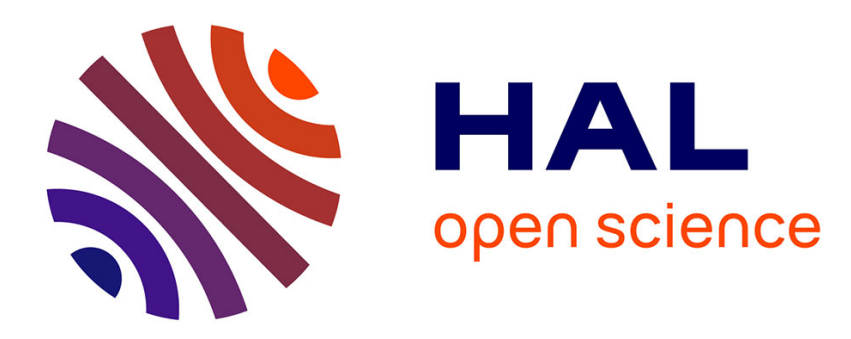

\title{
Troubles bipolaires et Rorschach : état de la question
} D. Fouques, H. Bénony

\section{To cite this version:}

D. Fouques, H. Bénony. Troubles bipolaires et Rorschach : état de la question. Pratiques Psychologiques, 2010, 16 (4), pp.375-388. 10.1016/j.prps.2009.05.002 . hal-02294170

\section{HAL Id: hal-02294170 \\ https://hal.parisnanterre.fr/hal-02294170}

Submitted on 23 Sep 2019

HAL is a multi-disciplinary open access archive for the deposit and dissemination of scientific research documents, whether they are published or not. The documents may come from teaching and research institutions in France or abroad, or from public or private research centers.
L'archive ouverte pluridisciplinaire $\mathbf{H A L}$, est destinée au dépôt et à la diffusion de documents scientifiques de niveau recherche, publiés ou non, émanant des établissements d'enseignement et de recherche français ou étrangers, des laboratoires publics ou privés. 


\title{
Troubles bipolaires et Rorschach : état de la question
}

\section{Bipolar disorders and Rorschach: State of the art}

\author{
D. Fouques ${ }^{\mathrm{a}, 1}$, H. Bénony ${ }^{\mathrm{b}, 2}$ \\ a Laboratoire Evaclipsy, université Paris-Ouest Nanterre-La Défense, 200, avenue de la République, \\ 92001 Nanterre cedex, France \\ ${ }^{\mathrm{b}}$ Laboratoire de psychopathologie et de psychologie médicale, pôle AAFE, université de Bourgogne, \\ BP 26513, 21065 Dijon cedex, France
}

\section{Résumé}

Cet article est une revue de la littérature sur l'usage du test de Rorschach dans le contexte des troubles bipolaires. Si les premiers travaux sur la question remontent aux origines même du test, il s'avère que peu d'études, à ce jour, ont été conduites sur ce sujet. Les recherches recensées concernent essentiellement : la description du fonctionnement psychologique des patients bipolaires dans les différentes phases de la maladie, la comparaison avec d' autres tableaux psychopathologiques à des fins de diagnostic différentiel (troubles unipolaires, schizophrénies, troubles schizoaffectifs) et la recherche d'endophénotypes. Les résultats de ces études sont résumés et leurs particularités méthodologiques discutées, ainsi que la pertinence du Rorschach comme outil d'investigation de cette pathologie.

Mots clés : Troubles bipolaires ; Rorschach ; Endophénotype

\begin{abstract}
This article is a review of the literature concerning the use of the Rorschach test in the context of bipolar disorders. Even though the first research dates back to the origin of the test, very few studies have been conducted so far. The known research mostly concerns: the description of the psychological functioning of bipolar patients throughout the different phases of the illness, the comparison with other psychopathological designs in order to set up a differential diagnosis (unipolar disorders, schizophrenic disorders, schizoaffective disorders) and to seek endophenotypes. This article aims at summarizing the findings of these studies and
\end{abstract}

\footnotetext{
1 Maître de conférence en psychologie clinique.

2 Professeur de psychologie clinique et de psychopathologie.
} 
at discussing their methodological specificities, as well as the relevance of the Rorschach test as a tool to investigate this pathology.

Keywords: Bipolar disorders; Rorschach; Endophenotype

\section{Introduction : problématiques psychologiques liées aux troubles bipolaires}

Les troubles bipolaires, actualisation du terme «psychose maniacodépressive », concernent aujourd'hui un nombre important de patients (prévalence de $1 \%$, voire plus selon les études), lesquels, en dépit de l'existence de traitements chimiques efficaces, souffrent encore de nombreuses difficultés, pendant et entre les phases aiguës de la maladie : risque suicidaire majeur, comorbidités anxieuses, addictives ou somatiques, stigmatisation sociale, symptômes résiduels et problèmes d'adaptation socioprofessionnelle. Leur origine biologique reconnue et l'existence de traitements chimiques thymorégulateurs relativement efficaces sont sans doute des facteurs qui peuvent expliquer l'intérêt limité que leur ont porté les psychologues jusqu'à une quinzaine d'années. Mais la perception des difficultés psychologiques et sociales susmentionnées a permis le développement d'études et de prises en charges psychologiques, aujourd'hui reconnues comme essentielles, en complément du traitement psychiatrique.

Parmi les problématiques intéressant les psychologues dans le champ de la bipolarité, nous retrouvons la question de l'évaluation à visée de contribution diagnostique. En effet, un des facteurs majeurs compliquant la prise en charge des patients souffrant de ce type de trouble est le retard au diagnostic, évalué à neuf ans en moyenne après le début des troubles. Comment expliquer ce retard? Plusieurs hypothèses sont possibles. Lors d'un début de trouble se manifestant sous l'angle dépressif, les spécialistes n'évoquent pas nécessairement la possibilité que cela entre dans le cadre d'une bipolarité. Il faut laisser le temps au caractère cyclique de la maladie d'apparaître. Par ailleurs, dans la forme dite de type II (dépression et hypomanie) où la phase d'excitation n'est pas aussi marquée que dans le type I (dépression et manie), les excitations hypomaniaques peuvent passer inaperçues, alors qu'aggravées par la prescription d'antidépresseurs, pourtant souvent proposés à ces patients considérés à tort comme souffrant de trouble unipolaire.

Une autre hypothèse serait qu'un début de maladie bipolaire très bruyant (phase maniaque avec des symptômes psychotiques) risque d'évoquer un trouble schizophrénique. Nombreux sont, en effet, les patients atteints de troubles bipolaires d'abord diagnostiqués schizophrènes. Précisons que seul $50 \%$ des patients bipolaires présentent des symptômes psychotiques durant les épisodes thymiques et que rares sont aujourd'hui les psychopathologues qui classent ce trouble parmi les psychoses (Pedinielli et Gimenez, 2004). Ce qui différenciera un trouble bipolaire d'un trouble schizoaffectif (ou schizophrénie dysthymique) et que, dans ce dernier cas, les symptômes psychotiques perdurent même en euthymie.

Enfin, les comorbidités anxieuses et addictives viennent compliquer le tableau symptomatique et certains patients alcoolodépendants, par exemple, une fois sevrés, laissent apparaître des symptômes de trouble bipolaire de type II jusqu'alors non repérés et donc non ou mal traités.

La question du diagnostic différentiel est donc très importante et concerne notamment la différenciation de cette affection avec les troubles unipolaires, schizophréniques et schizoaffectifs, mais aussi avec certains troubles de la personnalité (borderline, voire histrionique pour les principaux). 
En outre, les recherches actuelles en psychiatrie tendent à montrer la part génétique dans l'étiopathogénie de cette maladie. Pour préciser l'implication de ce déterminisme génétique, il est en premier lieu indispensable de bien caractériser la maladie sous les angles clinique, biologique, psychologique et neuropsychologique. Il s'avère qu' aujourd'hui tous les auteurs soulignent l'hétérogénéité de cette pathologie qui présente des formes cliniques variées, en fonction de différents facteurs : âge de début, présence ou non de symptômes psychotiques durant les épisodes thymiques, présence de comorbidité anxieuses et/ou addictives. . . autant de caractéristiques qui renvoient à des profils évolutifs et thérapeutiques différents, voire à des mécanismes étiopathogéniques particuliers. Ces profils différents ont encore besoin d'être précisés.

\section{Apport du Rorschach dans le champ de la bipolarité}

Nous nous sommes alors intéressés à la contribution du test de Rorschach dans le champ de cette pathologie afin de faire un état des lieux de ses éventuels apports dans le champ du diagnostic différentiel, d'une part, et de la description des différentes formes cliniques de cette maladie, d'autre part.

Partant de la description princeps, nous allons recenser les études Rorschach dans la maniacodépression, en tentant de les classer, ce qui est chose complexe. En effet, le but de ces études est souvent de mieux caractériser cette pathologie pour affiner le diagnostic et, notamment, le diagnostic différentiel entre troubles bipolaires versus troubles unipolaires ou encore versus schizophrénie et troubles schizoaffectifs, si bien qu'elles comportent souvent plusieurs groupes, de pathologies différentes, ce qui rend le regroupement thématique malaisé.

D'autres études ont tenté de comparer les profils Rorschach de bipolaires aux sujets tout venant, d'autres ont voulu comparer les particularités des bipolaires en fonction de leurs différents états thymiques. Enfin, quelques études ont voulu dégager des marqueurs de vulnérabilité dans une perspective génétique (recherche d'endophénotypes).

\subsection{Les premières études}

Hermann Rorschach (1921) s'intéresse déjà aux «maniaques dépressifs ». Dans son premier échantillon, quatre hommes et dix femmes portent ce diagnostic. Un sous-chapitre du Psychodiagnostik (inclus dans le chapitre sur les psychoses) traite de la «folie maniacodépressive » en montrant deux protocoles d'une même patiente, un premier pris en phase mélancolique, le second neuf mois plus tard, en phase maniaque.

Pour Hermann Rorschach, la variation maniaque de l'humeur représente le pôle opposé du syndrome dépressif. Les symboles qu'il utilise seront définis en termes psychologiques de l'époque, même s'ils sont aujourd'hui abandonnés ou modifiés. Dans l'hypomanie, il décrit que la vision des formes est altérée $\left(\mathrm{F}+\%^{3}\right)$ et plus encore dans la manie, la succession relâchée (l'ordre d'apparition des localisations) et le nombre de réponses globales (G) augmenté mais diminué dans la manie franche, le type d'appréhension (la proportion des réponses globales et de détails) est riche en hypomanie mais appauvri dans la manie. De même, la variabilité des contenus augmente dans l'hypomanie $\left(\mathrm{A} \%{ }^{4}\right)$ pour diminuer dans la manie, les réponses originales (orig ${ }^{5}$ )

\footnotetext{
3 Pourcentage de «bonnes formes ».

${ }^{4}$ Pourcentage de contenus animaux qui, pour Hermann Rorschach, lorsqu'il dépasse $50 \%$, renvoie à un fonctionnement stéréotypé et inférieur à $35 \%$ révèle des capacités d'imagination.

5 Soit les interprétations fournies environ une fois sur $100 \mathrm{chez}$ les normaux.
} 
sont plus nombreuses et de qualité médiocre, mais elles diminuent dans la manie franche. Le type de résonance intime (réponses $\mathrm{K}$ : kinesthésies humaines ou mouvements humains, comparées aux réponses $\mathrm{C}$ : couleurs) est dilaté en hypomanie pour se dilater encore plus lors de la manie. Le nombre de réponses est au-dessus de la moyenne chez l'hypomane et seulement un peu au-dessus chez le maniaque, ces derniers produisant beaucoup de réponses de contenu « objet». Les temps de réaction sont diminués, le $\mathrm{F}+\%$ est inversement proportionnel aux kinesthésies et les réponses humaines entières $(\mathrm{H})$ l'emportent sur celles de détail $(\mathrm{Hd})$ dans les deux cas.

En période dépressive ou mélancolique, la vision des formes est améliorée mais le reste du tableau est en faveur d'un appauvrissement de tous les facteurs et ce d'autant plus que l'humeur devient mélancolique.

Rorschach ne s'est intéressé majoritairement qu'à la dépression dans un contexte de «psychose maniacodépressive » et ne dit que peu de chose sur la «dépression psychogène », si ce n'est qu'il a eu l'occasion de l'observer chez des sujets qui produisant une à plusieurs kinesthésies, mais pas de réponses couleur.

Nous allons par la suite voir que Bhom se montre peut-être un peu enthousiaste quand il écrit :

«Bien que le matériel de Rorschach ne comportât que 14 patients maniaco-dépressifs, les syndromes que cet auteur a établis pour cette maladie font partie des acquisitions les plus sûres du test » (Bohm, 1955, p. 446).

Levey et Beck (1934), puis Beck et al. (1950) étudient au Rorschach des patients maniaques en les comparant à un groupe de sujets normaux et retrouvent un temps de réponse abaissé, une activité cognitive augmentée (Zf élevé et lambda bas) une réactivité à la couleur augmentée (WSumC), peu de réponse de relief provoqué par l'estompage (Vista), compris comme un manque d'introspection, ainsi qu'un $\mathrm{F}+\%$ et un nombre de réponses banales $(\mathrm{P})$ abaissé témoignant d'une insertion dans la réalité altérée traduisant, pour ces auteurs, le processus psychotique inhérent à cette pathologie, mais rien n'est décrit concernant les troubles de la pensée.

\subsection{Bipolaires, unipolaires et schizophrènes}

La première comparaison au Rorschach entre maniaques et schizophrènes est due à Schmidt et Fonda (1954). Dans cette étude comparative, en référence aux schizophrènes, les maniaques présentent une plus importante réponse émotionnelle (SumC augmentée) et produisent un effort cognitif plus important ( $\mathrm{Zf}$ augmenté et plus de réponses $\mathrm{M}^{6}$ ).

Ce sont Klopfer et Spiegelman (1956) qui commencent à repérer des différences sur la nature des réponses $\mathrm{M}-{ }^{7}$ produites par les maniaques par rapport à celles produites par des schizophrènes : ces derniers produisent des réponses à tonalité bizarre tandis que les maniaques génèrent ce type de réponses suite à des processus de balayage rapides et négligents, une composante impulsive dans le traitement de l'information.

Johnston et Holzman (1979) observent une quantité similaire de troubles de la pensée chez les maniaques et les schizophrènes, mais la nature de ces troubles est bien différente : les mécanismes combinatoires (associations ou mises en relations incongrues ou fantaisistes) sont le lot des maniaques. Cette étude a fait l'objet d'un suivi prospectif (Shenton et al., 1987 ; Solovay et al.,

\footnotetext{
${ }^{6} \mathrm{M}$ : mouvement humain : cotation américaine qui est à peu près l'équivalent de la $\mathrm{K}$ dans le système classique du Rorschach.

${ }^{7}$ Réponse de mouvement humain associée à une mauvaise forme.
} 
1987) qui confirme les données antérieures et précise quelques éléments qualitatifs concernant les productions maniaques combinatoires. Cette description sera reprise et complétée par Khadivi et al. (1997) et Petot (1999, 2003) qui insistent sur les caractéristiques ludiques, fantaisistes, désinvoltes et humoristiques des productions maniaques.

Donnelly et al. (1975) comparent 16 bipolaires et 13 unipolaires hospitalisés en phase de dépression. Ils différencient deux profils spécifiques : le style bipolaire serait caractérisé par une attention sélective aux aspects objectifs de la tache d'encre alors que les unipolaires auraient une approche plus subjective. De même, les réponses couleurs peu élaborées se retrouveraient uniquement chez les bipolaires.

Schaeffer (1977) compare au Rorschach 13 unipolaires, dix bipolaires et sept schizoaffectifs hospitalisés. L'humeur est mesurée durant l'évaluation. Celle-ci s'avère stable et constante durant la procédure (quatre évaluations), sans présenter de différences entre les trois groupes. Les cotations sont effectuées selon les critères d'Holtzman (1961) lesquels permettent notamment d'évaluer les troubles de la pensée (pathognomic verbalization) et le score de «niveau de développement ». On retrouve, d'ailleurs, dans ces huit critères d'Holtzman (fabulation, combinaisons fabulées, réponses bizarres, incohérence, logique autistique, contamination, détérioration de couleur et réponses absurdes) les ancêtres des six cotations spéciales cognitives du système intégré, dont la somme pondérée (WSUM6) mesure aujourd'hui les troubles de la pensée au Rorschach.

La « délimitation formelle» (form definiteness) correspond approximativement à ce qu'Exner définira comme la «qualité de développement»(DQ). Elle se cote sur une échelle allant de 0 à 4 . Un « 0 » est attribué pour les réponses «amorphes» (ex : nuage, du sang), équivalent strict de la qualité de développement vague - DQv - en système intégré (SI). Les autres catégories traduisent une plus grande organisation cognitive du percept, sans que l'on puisse facilement les faire correspondre aux actuels critères de cotation de la DQ en système intégré.

Les résultats font état d'un plus grand nombre de troubles de la pensée chez les bipolaires et les schizoaffectifs que chez les unipolaires, sans différence entre les bipolaires et les schizoaffectifs.

En ce qui concerne la «délimitation formelle», les unipolaires ont des résultats supérieurs aux schizoaffectifs mais comparables à ceux des bipolaires. La différence entre bipolaires et schizoaffectifs montre une tendance à la significativité $(p<0,10)$.

Aucune corrélation n'est retrouvée entre les scores de troubles de la pensée et ceux de délimitation formelle.

Pour résumer, l'intérêt de cette étude est de montrer la similitude entre les bipolaires et les unipolaires en ce qui concerne l'adaptation sociale et le degré de sophistication des formes produites au Rorschach, ainsi que leur différence concernant les troubles de la pensée, plus importants chez les bipolaires, à un niveau de ceux présents chez les schizoaffectifs ou tout juste inférieur.

Mormont et al. (1990) partent des deux études précitées (Schaeffer, 1977 ; Donnelly et al., 1975) dans une optique de réplication. Quatorze unipolaires et 12 bipolaires sont testés en phase de dépression sévère (humeur mesurée et comparable entre les deux groupes).

Face à l'absence d'homogénéité des systèmes de cotation, les auteurs, utilisant le système intégré, assimilent les «réponses amorphes» au DQv et les «pathognomic verbalization» au WSUM6. Ils confirment un seul résultat de Donnelly : la présence de DQv en plus grande quantité chez les bipolaires et le résultat de Schaeffer qui observe des troubles de la pensée en plus grand nombre chez les bipolaires. Ils insistent sur le fait que la différence n'est pas apparente si on compare les moyennes des scores du WSUM6 dans les deux groupes (approche dimensionnelle) mais qu'elle apparaît $(p=0,04)$ si on compare le pourcentage de sujets obtenant un score WSUM6 supérieur ou égal à 7 (approche catégorielle). 
«Il semblerait que l'on observe plus souvent chez les bipolaires un moment de relâchement de l'effort de synthèse perceptive (d'où plus de réponses amorphes) et des processus de pensée (d'où plus de verbalisations inhabituelles)» p. 218.

Ils insistent sur le fait que $50 \%$ des sujets de chacun des groupes ne présentent pas les caractéristiques mesurées en dépit de la significativité :

«[...] schématiquement, la moitié des unipolaires présentent une caractéristique que ne présentent que peu de bipolaires (en l'occurrence $D Q v=0)$ et vice versa $(D Q v>3$ et WSUM6 $\geq 7$ ). Autrement dit, les originalités qui spécifient les deux échantillons ne permettent de ne décrire que la moitié de chacun d'eux... [...] Nous dirons donc que le fait de ne jamais donner un signe de relâchement de l'exigence formelle (dans le sens de «forme définie» et non pas de celui de «forme exacte») caractérise la moitié des unipolaires - et eux seuls-alors que les indices d'un grand laxisme sur ce plan et/ou sur celui des processus de la pensée se rencontrent chez la moitié des bipolaires - et eux seuls. » p. 215.

Par ailleurs, les résultats tendent à montrer l'absence d'homogénéité des protocoles de Rorschach de déprimés (indépendamment du caractère uni- ou bipolaire de la dépression) et les auteurs invitent à la prudence concernant l'utilisation classique de regroupement de signes, soi-disant typiques de la dépression au Rorschach, lesquels sont proposés à la fois en méthode classique française et d'autres en système intégré (constellation DEPI). En effet, aucun de leurs sujets ne présente une constellation DEPI positive, pourtant supposée repérer la dépression, au moins en population américaine. Les auteurs préconisent alors un usage circonspect de cette constellation dans les pays francophones.

La discussion s'amorce ensuite sur le débat psychopathologique suivant: l'absence d'homogénéité des protocoles semble indiquer que la dépression, qu'elle soit uni- ou bipolaire, peut se manifester sur tout type de personnalité, à laquelle «elle n'imprime pas une marque uniforme » (p. 216). Ils discutent alors la notion de «personnalité dépressive » (entité présente dans le DSM-III, à l'époque de l'étude, aujourd'hui disparue du DSM-IV) pour décrire que cette notion recouvre, en fait, deux réalités cliniques différentes. Une première qui décrirait des personnalités marquées par des traits dépressifs (pessimisme...) sans pour autant qu'elle indique un risque de décompensation vers une «dépression», une seconde caractérisée par une personnalité dont la configuration et le fonctionnement «conduit à la dépression (comme par exemple, le font des exigences excessives du moi idéal)» (p. 217).

D'après ces auteurs, seule la première serait repérable au Rorschach. Ils appliquent le même raisonnement à la manie qui serait soit « structurelle», soit un avatar de l'expérience, sans s'inscrire dans un fonctionnement stable de la personnalité.

Si $50 \%$ des protocoles maniaques présentent des particularités, la méthodologie employée ne permet pas de dire si ces éléments apparaissent comme «résiduels » des épisodes maniaques ou s'ils sont présents en amont.

Enfin, les auteurs considèrent que les réponses «amorphes » et les «verbalisations inhabituelles » peuvent être comprises, si retrouvées chez un déprimé, comme marqueur d'un épisode maniaque antérieur ou d'un risque de développer plus tard une manie (seul un suivi prospectif après un premier épisode non maniaque permettrait de le savoir). Ils l'expliquent en revenant aux particularités cliniques de la manie (présence de processus primaires et fautes logiques) pour justifier les troubles de la pensée et relâchement perceptif dus à l'exaltation, l'hypersensorialité et la facilité d'association pour expliquer les réponses vagues.

Les auteurs concluent en insistant sur la nécessité d'évaluer ces patients en phase de normothymie, afin de séparer ce qui relèverait d'un trait ou d'un état, et sur la pertinence de tester de 
manière prospective des patients avant un premier épisode maniaque pour statuer sur l'origine phénoménologique ou structurale des particularités observées.

\subsection{Les profils bipolaires en fonction de l'état thymique}

Une seule étude à notre connaissance compare les mêmes 15 patients bipolaires en phase maniaque et dépressive, avec mesure des symptômes thymiques au Rorschach (et au Hand test) (Wagner et Heise, 1981). Les groupes sont comparés à l'aide du test de Wilcoxon utilisé sur les médianes. Les différences significatives observées concernent :

- la somme des couleurs (SumC) : la médiane passant de 8 en phase maniaque à 0 en phase dépressive;

- le F\% (le nombre de réponses uniquement formelles du protocole divisé par le nombre total de réponses, soit approximativement l'équivalent du lambda en SI) : passant de 36 en manie à 89 en dépression;

- le $\mathrm{A} \%$ : (pourcentage de réponses à contenu animal) : passant de 43 en manie à 67 en dépression.

Ces résultats confirment partiellement les premières observations d'Hermann Rorschach.

Singer et Brabender (1993) tentent de repérer des différences entre patients unipolaires et bipolaires au Rorschach (SI). L'étude consiste en la comparaison de trois groupes de patients : 29 unipolaires déprimés, 15 bipolaires déprimés et 18 bipolaires maniaques. Leurs résultats montrent des différences entre les groupes au niveau de deux secteurs du fonctionnement cognitif : l'idéation (mode de conceptualisation, de construction des représentations mentales) et la médiation cognitive (qualité de l'adéquation entre le percept et la découpe objective du stimulus, ou testing de la réalité). Nous résumons les résultats discriminant les trois groupes et ceux discriminant les deux groupes bipolaires du groupe unipolaire.

\subsubsection{L'idéation}

Pour les variables dimensionnelles, le SUM6 et le WSUM6 différencient nettement les trois groupes (Anova vérifiée a posteriori pour chaque groupe deux à deux à $p=0,05$ par comparaison de Newman-Keuls) (Tableau 1).

Pour les variables catégorielles, à l'aide du test de $\mathrm{Chi}^{2}$ corrigé pour les petits effectifs, la présence de réponse déviantes (DR) de niveau 1 et la présence de plus d'une combinaison incongrue (INC) de niveau 1 différencient les trois groupes ${ }^{8}$ (Tableau 2 ).

\subsubsection{La médiation cognitive}

Seule la variable $\mathrm{X}-\%$ (soit le pourcentage de réponses dont la qualité formelle est mauvaise) s'avère différente entre les bipolaires et les unipolaires (même méthode de vérification statistique que précédemment) (Tableau 3).

Outre ces résultats dans la sphère cognitive, d'autres paraissent intéressants. En effet, la constellation DEPI ne repère que $26 \%$ des bipolaires déprimés, 59 \% d'unipolaires déprimés et s'avère

\footnotetext{
${ }^{8}$ DR et INC sont deux des six cotations spéciales dont la somme pondérée, en fonction de critère de gravité (niveau 1 : léger à $2:$ grave) constitue une mesure des troubles de la pensée au Rorschach (WSum6). DR est coté lorsque le sujet ajoute à sa réponse un commentaire «à côté », inapproprié, pouvant aller jusqu'à une fuite des idées (ex : un papillon, ma femme en a très peur !). INC est coté lorsque que le sujet associe ou juxtapose différentes parties de la planche en un seul objet, présentant alors des caractéristiques qui ne lui sont pas propres (ex : planche I : une chauve-souris avec des mains).
} 
Tableau 1

Comparaison des scores de troubles de la pensée, d'après Singer et Brabender (1993): approche dimensionnelle.

\begin{tabular}{|c|c|c|c|c|c|c|c|c|}
\hline & \multicolumn{2}{|c|}{ Bipolaires maniaques } & \multicolumn{2}{|c|}{ Bipolaires déprimés } & \multicolumn{2}{|c|}{ Unipolaires déprimés } & \multicolumn{2}{|c|}{ Tests Statistiques } \\
\hline & M & S.D. & M & S.D. & M & S.D. & $F$ & $p$ \\
\hline SUM6 & 6,94 & 4,05 & 3,73 & 2,37 & 1,69 & 1,65 & 20,84 & 0,00001 \\
\hline WSUM6 & 26,28 & 15,62 & 12,07 & 9,07 & 5,83 & 7,32 & 20,26 & 0,00001 \\
\hline
\end{tabular}

Tableau 2

Comparaison des scores de troubles de la pensée, d'après Singer et Brabender (1993) : approche catégorielle.

\begin{tabular}{|c|c|c|c|c|c|}
\hline & $\begin{array}{l}\text { Bipolaires } \\
\text { maniaques }(\mathrm{BM}) \\
\%\end{array}$ & $\begin{array}{l}\text { Bipolaires } \\
\text { déprimés (BD) } \\
\%\end{array}$ & $\begin{array}{l}\text { Unipolaires } \\
\text { déprimés (UD) } \\
\%\end{array}$ & $\begin{array}{l}\mathrm{BM} \text { vs UD } \\
p\end{array}$ & $\begin{array}{l}\text { BD vs UD } \\
p\end{array}$ \\
\hline $\mathrm{DR}>0$ & 77 & 60 & 14 & 0,000 & 0,001 \\
\hline INC > 1 & 50 & 36 & 03 & 0,022 & 0,000 \\
\hline
\end{tabular}

Tableau 3

Comparaison des scores du testing de la réalité (X-\%), d'après Singer et Brabender (1993).

\begin{tabular}{|c|c|c|c|c|c|c|c|c|}
\hline & \multicolumn{2}{|c|}{ Bipolaires maniaques } & \multicolumn{2}{|c|}{ Bipolaires déprimés } & \multicolumn{2}{|c|}{ Unipolaires déprimés } & \multicolumn{2}{|c|}{ Tests Statistiques } \\
\hline & M & S.D. & M & S.D. & M & S.D. & $F$ & $p$ \\
\hline $\mathrm{X}-\%$ & 0,23 & 0,14 & 0,21 & 0,09 & 0,14 & 0,08 & 5,19 & 0,008 \\
\hline
\end{tabular}

positive chez $37 \%$ des maniaques (la différence est significative à 0,046 uniquement entre déprimés uni- et bipolaires). Ces résultats confirment et étendent les doutes de Mormont et al. (1990) sur la validité de cette constellation censée repérer les dépressions. La constellation $\mathrm{SCZI}^{9}$, censée à l'époque de l'étude repérer des caractéristiques schizophréniques mais aujourd'hui abandonnée, est positive chez $33 \%$ des patients maniaques versus $0 \%$ chez les bipolaires déprimés et $3 \%$ chez les unipolaires déprimés (différence significative uniquement entre bipolaires maniaques et déprimés à 0,02 , et entre maniaques et unipolaires à 0,005). Enfin, les bipolaires déprimés présentent un niveau bas d'activité d'organisation perceptive et de mise en relation des percepts, comparativement aux unipolaires.

Les auteurs concluent:

«lorsque la dépression est manifeste (à travers le Rorschach ou tout autre mode d'observation) et qu'un score particulièrement élevé de cotations spéciales est repéré, d'autant plus qu'il s'associe à un déficit du testing de la réalité, l'hypothèse d'un trouble bipolaire doit être posée » p. $343^{10}$.

En effet, les variables Rorschach suivantes : SUM6, WSUM6 (dont DR $>0$ et INC $>1$ ) et X-\% élevés, retrouvées chez un patient déprimé, doivent permettre d'évoquer une bipolarité.

En dépit de qualités méthodologiques certaines, nous repérons quelques limites à cette étude : l'absence de contrôle de l'impact de l'intensité des symptômes thymiques sur les variables Rorschach. Cela semble dû à la taille des échantillons qui n'autorise pas des analyses statistiques

\footnotetext{
${ }^{9}$ Ancêtre de l'actuelle constellation PTI qui évalue les troubles de la pensée et de la perception.

10 Traduction personnelle.
} 
multivariées. Les auteurs eux-mêmes suggèrent d'inclure des patients euthymiques dans des recherches futures pour repérer quelles sont les variables stables. Enfin, nous ne connaissons pas les antécédents, notamment maniaques, des patients bipolaires déprimés, ni quelle était l'intensité de la manie, autant de facteurs qui pourraient pourtant influencer les données. De plus, les sujets étaient aptes à être testés, donc ne devaient pas être dans une phase maniaque très sévère, d'autant plus qu'ils sont testés au plus tard dix jours après leur admission à l'hôpital.

Weiner (2003) fait une synthèse des signes retrouvés dans la manie et Castro (2006), en se référant à ces travaux, en précise le cadre :

«Dans la psychiatrie contemporaine, la manie est devenue un concept complexe. Elle est incluse dans la notion d'état mixte (états mixtes déjà isolés par Kraeplin), notion qui reconnaît le fait que la manie ne peut être simplement un état euphorique ou expansif, mais aussi un état aigu, infiltré d'angoisse. L'euphorie, le sentiment de toute puissance, les idées de grandeur ont un impact notable sur l'ensemble du fonctionnement psychique individuel qui réagit ainsi à une situation de perte ou qui lutte de cette manière contre l'angoisse (Caillard, 1982). » (Castro, 2006, p. 156).

Même si beaucoup de psychopathologues distinguent cependant «états mixtes » et «états maniaques », cette conception vient en écho à l'idée, chère à Henry (2005), que la manie n'est pas synonyme d' euphorie et que bien des états émotionnels s'y retrouvent, notamment des affects de type dépressif. Ce point de vue rejoint la conception psychanalytique de la manie comme défense contre la dépression, qu'elle n'arrive cependant pas à masquer totalement, puisqu'elle est apparente dans les protocoles de Rorschach, même en phase maniaque.

Castro (2006) reprend les signes de Weiner lequel «s'attache aux formes mixtes de la manie, qui supposent la coexistence des critères de la série dépressive et maniaque » (ibid. p. 156), en les classant en variables affectives (registres dépressifs et maniaques) et cognitives.

Weiner décrit une positivité de la constellation DEPI (malgré les réserves précédemment émises), des affects pénibles intériorisés (excès de $C^{\prime}$ ), la présence de confusion, d'ambivalence affective (blends couleur-estompage) dans le registre dépressif. Dans le registre maniaque, apparaissent des difficultés à moduler les émotions $(\mathrm{CF}+\mathrm{C}>\mathrm{FC})$, une alternance de contenus à valences opposées, tantôt optimistes, tantôt pessimistes, et une expression labile et exagérée des émotions. Au plan cognitif, une perturbation du sens discriminatoire est décrit (INC), ainsi qu'une incapacité à prendre du recul, un débordement par les stimulations (lambda bas), une pensée hermétique et incohérente (présence DR1 et DR2) et une complexité psychique augmentée (blends très complexes en grand nombre).

Cette synthèse récente s'intéresse donc aux patients maniaques, considérés comme des déprimés latents en train de s'épuiser pour lutter contre la perte dépressive.

\subsection{Des études originales}

Une étude (MacHovec, 1982), propose une analyse psycholinguistique des productions au Rorschach. Trois groupes de sujets sont définis en fonction d'un diagnostic psychiatrique et des données du Rorschach :

- groupe 1 :troubles de la pensée psychotiques de types schizophréniques (46 sujets);

- groupe 2 : troubles affectifs avec des symptômes psychotiques (31 sujets);

- groupe 3 : troubles non psychotiques (46 sujets). 
Sont étudiées les formes contentives des verbes et des noms ainsi que les activations ou non des objets animés ou inanimés.

Les mots (noms, verbes ou adjectifs) sont contentifs dès lors qu'ils contiennent un sens (les mots : de, la, sur, entre, etc. ne sont pas des contentifs). L'auteur attire notre attention sur le fait qu'un objet grammaticalement inanimé (ex : nuage) peut être «activé » ou «émotionalisé » par un modificateur de type forme verbale contentive (ex : nuage lent et menaçant).

Les résultats concluent que les patients du groupe 1 (type schizophrénique) ont tendance à déconnecter le mouvement ou l'émotion du percept, à «dés-animer» des objets qui sont grammaticalement animés, alors que les sujets psychotiques du groupe affectif (2) tendent à faire l'inverse. Quant aux non psychotiques, ils utilisent un mélange plus équilibré de ces deux modes. Cette méthode permettrait alors de discriminer ces deux formes de troubles avec des éléments psychotiques, mais cette étude n'a pas fait d'émules.

Mormont (1983), propose une lecture qualitative phénoménologique d'un protocole de patient bipolaire au décours d'un épisode maniaque et souligne la bipolarité des contenus selon des axes qu'il définit : haut et bas, ciel et terre, léger et lourd, vie et mort, animé et dévitalisé, dense et inconsistant. Il montre ainsi que la «bipolarité » ne s'exprime pas uniquement dans le registre de la thymie (cf. Weiner, 2003), dans la continuité des idées de Kraeplin.

Wagner et Rinn (1994) pensent avoir repéré un signe pathognomonique de manie au Rorschach: the extrapolated area response (EAR). L'EAR est cotée quand un sujet identifie une partie d'un percept dans une partie de la tache puis, pour compléter l'image, étend le contour de l'objet à l'extérieur de la tache, dans l'espace blanc, voire au-delà des limites de la planche. Ce comportement, traduisant une projection singulière plus qu'une hallucination, est donné par les patients maniaques, sans aucune conscience de la prise de liberté qu'ils s'accordent. Ils se montreraient assez satisfaits de ce qu'ils semblent parfois considérer comme une réponse d'une qualité supérieure. Les auteurs interprètent ce signe comme l'expression de la «tendance maniaque à s'épandre au-delà des limites de la réalité et d'entreprendre des exploits qui vont inévitablement échouer dans le monde externe » (ibid. p. 1026) ${ }^{11}$. Les auteurs n'ont retrouvé ce signe qu'en phase maniaque, dans leur échantillon précédent (Wagner et Heise, 1981) et invitent à une validation plus ample, en testant notamment des sujets en élation de l'humeur induite par des substances ou lors d'hypomanies.

Plus récemment, et dans une perspective psychodynamique, Chabot et al. (2003), à partir d'une analyse langagière des productions de patients bipolaires au Rorschach (ainsi qu' au TAT), mettent en évidence deux dimensions : la référence à l'intensité, se manifestant dans le langage, l'autre caractérisant le type d'angoisse renvoyant à la persécution, indépendamment de la grande diversité d'organisations psychiques soulignée parmi les huit patients testés.

Cette diversité de fonctionnement psychique fait écho au travail de Sagelas-Granval (2004) qui retrouve dans des protocoles de Rorschach de patients bipolaires euthymiques, des éléments évocateurs d'un fonctionnement limite.

\subsection{La recherche de marqueurs de vulnérabilité}

Concernant la recherche de marqueurs de vulnérabilité chez les apparentés, Worland (1979) étudie les données Rorschach d'enfants de bipolaires et de schizophrènes, en s'intéressant au «niveau de développement ${ }^{12}$ (developmental level [DL]). Il étudie 305 enfants âgés de six

\footnotetext{
11 Traduction personnelle.

12 Un indice qui n'est plus utilisé et qui s'approcherait de la qualité de développement (DQ) en système intégré.
} 
à 20 ans : 79 issus d'un couple dont un des parents est schizophrène, 51 d'un couple dont un est maniacodépressif, 59 ont un parent souffrant de maladie physique et 116 témoins. L'âge, le statut socioéconomique et l'intelligence (QI obtenu aux échelles de Wechsler) sont traités comme covariables.

Le score au DL est significativement plus bas chez les enfants de schizophrènes que chez tous les autres. Aucune autre différence n'est retrouvée entre les autres groupes. Les enfants de bipolaires sont parfaitement comparables aux témoins. Par ailleurs, les différences observées, bien que significatives, sont très faibles et l'auteur conclut qu'en aucun cas ce score ne doit être considéré comme marqueur de vulnérabilité.

Mandel et al. (1984) ont testé au Rorschach 35 patients bipolaires euthymiques afin de repérer des marqueurs génétiques éventuels. Cette étude se limite à la comparaison des résultats obtenus aux normes américaines d'Exner (1978) et n'a pas de groupe témoin. L'euthymie est opérationnalisée par: l'avis du psychiatre référent, l'absence de signes moteurs dans les entretiens psychiatriques, l'absence de signes thymiques et végétatifs depuis au moins deux semaines et l'avis de la famille sur l'état fonctionnel du patient ainsi que l'absence d'épisode depuis au moins un mois. Par ailleurs, les patients sont précisément décrits au plan des caractéristiques cliniques de la maladie (nombre d'hospitalisations, nature des précédents épisodes, traitements au moment du Rorschach).

Les auteurs s'intéressent alors aux neuf variables Rorschach déviant d'un écart-type des données normatives pour $50 \%$ des sujets de leur échantillon. Dans l'ordre de la plus importante déviance vers la moindre, sont retrouvées les variables suivantes (inférieures aux normes): les réponses $\mathrm{FC}$, la qualité formelle $(\mathrm{F}+\%)$, le $\mathrm{Zf}$ (effort cognitif lors du traitement perceptif), les blends (la complexité psychique), les réponses humaines réelles entières et partielles $(\mathrm{H}+\mathrm{Hd})$, les mouvements humains, les nombres de réponses basées sur la symétrie (paires) et le pourcentage de réponses animales (A\%). En revanche, les bipolaires présentent une proportion plus importante de troubles de la pensée (ancêtre du WSUM6) par rapport aux normes. Ces éléments décrivent alors chez les bipolaires euthymiques une difficulté de modulation des affects, un testing de la réalité altéré, une activité cognitive moindre, des compétences relationnelles affectées et des troubles de la pensée en nombre important comparativement aux non consultants américains. Les auteurs ne retrouvent pas de différences en fonction du sexe, de l'âge et du niveau d'étude. Ils concluent alors que ces variables pourraient être testées comme marqueurs endophénotypiques éventuels.

Poursuivant le même objectif, Last et al. (1989) s'intéressent aux potentialités du Rorschach pour repérer des marqueurs de vulnérabilité génétique, en comparant les résultats au Rorschach de 35 patients bipolaires euthymiques aux données normatives (l'étude précitée de Mandel et al., 1984) et les résultats au Rorschach de 20 enfants non consultants de bipolaires à des témoins appariés.

En conservant les valeurs Rorschach présentant des différences entre les deux échantillons et le groupe témoin ou les normes, les auteurs définissent alors les marqueurs de vulnérabilité génétiques potentiels suivants: $\mathrm{Zf}, \mathrm{FC}, \mathrm{W}, \mathrm{Dd}$, lambda, A\%, FQF+, P, H et $\mathrm{Hd}$ abaissés, et SUMS (équivalent de WSUM6) augmenté. Ces éléments renvoient à une faiblesse de l'activité organisationnelle et de synthèse, un évitement de la complexité, un testing de la réalité altéré, des troubles de la pensée et des particularités négatives des relations. Les résultats s'avèrent discutables à plus d'un titre. L'étude reprise de Last présente les mêmes lacunes, mais des résultats détaillés y sont présentés alors qu'ils ne l'étaient pas dans la première version. Par ailleurs, la diminution des scores à certaines variables isolées ne permet pas l'interprétation que les auteurs en donnent: en effet, si le FQF+ (nombre de bonnes formes) est plus bas chez les bipolaires euthymiques et les patients, est-ce au profit de formes mauvaises et/ou originales ? Les données ne le mentionnent pas. 
Une étude plus convaincante issue de la même équipe de recherche (Osher et al., 2000), se focalise uniquement sur un groupe de 14 enfants sains de parents bipolaires comparé à un groupe de 14 enfants sains et sans antécédents. Ils constatent dans le groupe test une diminution du nombre de FC, de $\mathrm{M}$ et de $\mathrm{P}$ et une augmentation des cotations spéciales de niveau 2 (Lvl2) (en moyenne et non en fréquence), une augmentation du WSUM6 (6,14 vs 2,93), et une augmentation du nombre de sujets présentant la constellation $\mathrm{CDI}^{13}$ positive $(71,4 \%$ vs $35,7 \%)$. Ces variables sont alors proposées comme marqueurs potentiels de vulnérabilité.

Récemment, Osher et Bersudsky (2007), dans la continuité des travaux précédents, comparent les Rorschach de 18 patients adultes bipolaires euthymiques à huit sujets témoins appariés selon l'âge et le sexe. Ils retrouvent surtout une augmentation du WSUM6 (12,83 vs 5,5) et du A\% $(43,5$ vs 34,37$)$ chez les bipolaires et une diminution du nombre de réponse FC (1,67 vs 3,12$)$. Les auteurs insistent sur le potentiel de ces trois variables (troubles de la pensées, manque de réponses émotionnelles «secondarisées» et contenus immatures) comme marqueurs endophénotypiques.

\section{Synthèse et perspectives}

L'intérêt pour les troubles bipolaires au Rorschach, bien que remontant aux origines même du test, n'a pas suscité beaucoup de travaux de recherche.

Pourtant, les quelques études recensées mettent en évidence des différences dans les profils Rorschach entre les différentes phases de la maladie, mais aussi entre la maniacodépression et d'autres pathologies : troubles schizophréniques, schizoaffectifs et troubles unipolaires. Ce dernier point souligne la pertinence du Rorschach pour contribuer au diagnostic différentiel mais des approfondissements sont nécessaires. En effet, peu d'études de réplication ont été effectuées, peutêtre est-ce dû en partie à l'absence de consensus sur les systèmes de cotation et d'interprétation. Il serait donc hasardeux de tirer des conclusions généralisables, ce d'autant que les études comportent souvent des biais, du fait d'échantillons de faible taille: un manque de puissance statistique et du fait d'une absence de prise en compte au plan statistique de l'influence de l'état thymique des patients sur leurs productions au Rorschach, même s'il est parfois évalué.

Peu de données sur les particularités des bipolaires en phase intercritique sont disponibles, en dépit de l'invitation des auteurs à développer ces études, afin de repérer d'éventuelles caractéristiques stables. Mais on sait aujourd'hui que normothymie ne veut pas dire absence de symptômes et que les symptômes dits résiduels ont un impact important dans le fonctionnement des bipolaires pourtant euthymiques.

Par ailleurs, les variables Rorschach semblent fluctuer beaucoup chez un même patient déprimé ou maniaque, on le sait depuis Hermann Rorschach. Il y a donc des raisons de faire l'hypothèse que, même entre les épisodes thymiques, des symptômes résiduels peuvent avoir une influence sur leur profil Rorschach.

La question de la présence de troubles de la pensée (idéation) et, dans une moindre mesure, de la perception (médiation) a permis de montrer qu'ils n'étaient pas propres aux schizophrènes et qu'ils sont aussi présents chez les bipolaires (comme chez les unipolaires déprimés), même en euthymie, malgré la différence de nature et la variation de l'intensité avec l'état thymique.

Enfin, il n'a pas été possible de dégager un profil type de bipolarité au Rorschach. Même si des valeurs de groupes permettent de montrer des différences, il n'en demeure pas moins que l'absence d'homogénéité des profils suggère l'existence de sous-groupes parmi les bipolaires. Quant à la

\footnotetext{
${ }^{13}$ Le CDI reflète un déficit de compétences relationnelles et une grande vulnérabilité au stress.
} 
recherche de marqueurs de vulnérabilité, certaines études laissent penser que le Rorschach pourrait être d'un apport notable dans ce champ et méritent d'être répliquées.

Enfin, aucune étude à ce jour n'a permis de faire le distinguo entre des caractéristiques Rorschach «structurelles » à la bipolarité (préexistant au déclenchement du trouble) et des empreintes laissées dans le fonctionnement psychique par la pathologie (symptômes psychotiques, nombre d'épisodes, âge de début...).

Cependant, nous retrouvons dans la plupart des études une particularité : l'élévation des troubles de la pensée, présente même chez les malades en phase aiguë, en euthymie et chez des apparentés sains. Ils pourraient alors se rapprocher des critères requis pour être un marqueur de vulnérabilité, comme l'ont suggéré certains auteurs, voire un marqueur endophénotypique.

Soulignons, enfin, la nécessité de prudence dans l'usage de la constellation DEPI qui, aujourd'hui tend à être interprétée comme témoin d'une vulnérabilité affective plutôt que comme reflet d'un état symptomatique dépressif, et ce d'autant plus quand son score est bas (Andronikof et Réveillère, 2004).

Ces travaux permettent de penser que le Rorschach peut contribuer à mieux cerner les troubles bipolaires, que ce soit sous l'angle du diagnostic différentiel, ou du démembrement de l'hétérogénéité du trouble (définition de sous-groupes et de son étiopathogénie notamment génétique). Cette contribution nécessite alors de poursuivre l'étude de ces patients, notamment en période d'euthymie, mais en considérant des variables telles que les symptômes résiduels et les caractéristiques cliniques de la maladie (nombre et nature des épisodes, présence ou absence de symptômes psychotiques durant les phases...). En effet, ces variables pourraient avoir une influence sur les caractéristiques psychologiques repérées au Rorschach.

\section{Conflits d'intérêts}

\section{Aucun.}

\section{Références}

Andronikof, A., Réveillère, C., 2004. Rorschach et psychiatrie : à la découverte du malade derrière la maladie. Psychologie Française 49, 95-110.

Beck, S.J., Rabib, A.L., Thiesen, W.G., Molish, H., Theftford, W.N., 1950. The normal personality as projected on the Rorschach. Journal of Psychology 30, 241-298.

Bohm, E., 1955. Traité du psychodiagnostic de Rorschach. PUF, Paris.

Castro, D., 2006. Pratique de l'examen psychologique en clinique adulte. Dunod, Paris.

Chabot, M., Husain, O., Reeves, N., Choquet, F., 2003. La maladie bipolaire au Rorschach et au TAT: diversité ou homogénéité ? Diagnostics et pronostics. Psychologie Clinique et Projective 9, 255-283.

Exner, J.E., 1978. The Rorschach: a comprehensive system. II. Current research and advanced interpretation. New York: Wiley.

Donnelly, E. F., Murphy, D. L., Scott, W. H., 1975. Perception and cognition in patients with bipolar and unipolar depressive disorders. A study in Rorschach responding. Archive of General Psychiatry, 32(9), 1128-31.

Henry, C., 2005. Clinique des troubles bipolaires. In: Leboyer, M. (Ed.), Troubles bipolaires : pratiques, recherches et perspectives. John Libbey Eurotext, Paris, pp. 9-25.

Holtzman, W.H., 1961. Guide to administration and scoring: Holtzman Inkblot Technique. New York: Psychological Corp.

Johnston, M., Holzman, P.S., 1979. Assessing Schizophrenic Thinking. Jossey-Bass, San Fransisco.

Khadivi, A., Wetzler, S., Wilson, A., 1997. Manic indices on the Rorschach. Journal of Personality Assessment 69, 365-375.

Klopfer, B., Spiegelman, M., 1956. Differential diagnosis. In: Klopfer, B., Ainsworth, M., Klopfer, W., Holt, R. (Eds.), Development in Rorschach Technique II: Field Application. World Book, Yonkers-on-Hudson, NY, pp. 281-317. 
Last, U., Mandel, B., Shapiro, E., Belmaker, R.H., 1989. In search of psychological markers of bipolar manic-depressive illness (BMDI): commonalities in psychological functioning between adult euthymic BMDI patients and offspring of BMDI-affected parents. Israelian Journal of Psychiatry and Related Science 26, 75-84.

Levey, D.M., Beck, S.J., 1934. The Rorschach test in manic-depressive psychosis. Research Publication of the Association for Nervous and Mental Disorders 4, 31-42.

MacHovec, F.J., 1982. Differantiating affective from though disorders by semantic analysis of Rorschach responses. Journal of Personality Assessment 46, 12-17.

Mandel, B., Last, U., Belmaker, R.H., Rosenbaum, M., 1984. Rorschach markers in euthymic manic-depressive illness. Neuropsychobiology 12, 96-100.

Mormont, C., 1983. Quelques expressions de la bipolarité maniacodépressive au Rorschach. Les Feuillets Psychiatriques de Liège 16, 37-40.

Mormont, C., Andronikof-Sanglade, A., Vermeylen-Titron, N., Pardoen, D., 1990. Comparaison de déprimés unipolaires et bipolaires au moyen du Rorschach (comprehensive system d'Exner). Revue de Psychologie Appliquée, $2^{\mathrm{e}}$ trimestre, 40, 207-218.

Osher, Y., Mandel, B., Shapiro, E., Belmaker, R.H., 2000. Rorschach markers in offsprings of manic-depressive patients. Journal of Affective Disorders 59, 231-236.

Osher, Y., Bersudsky, Y., 2007. Thought disorder in euthymic bipolar patients. A possible endophenotype of bipolar affective disorder? The Journal of Nervous and Mental Disease 195, 857-860.

Pedinielli, J.L., Gimenez, G., 2004. Les psychoses de l'adulte. Armand Colin, Paris.

Petot, D., 1999. Mania and thought disorders in ten-year-old boy. Rorschachiana Yearbook of the international Rorschach Society $24,58-75$.

Petot, D., 2003. Hypomanie, manie, troubles bipolaires et cyclothymie. In: Petot, D. (Ed.), L'évaluation clinique en psychopathologie de l'enfant. Dunod, Paris, pp. 275-276.

Rorschach, H., 1921. Psychodiagnostic, $7^{e}$ édition. PUF, Paris (1993).

Sagelas-Granval, C., 2004. Les troubles bipolaires : réflexions psychopathologiques et perspectives psychothérapeutiques. Perspectives Psy, 43, 387-403.

Schaeffer, D. S., 1977. Scores on neuroticism pathology, mood, and Rorschach and diagnosis of affective disorder. Psychological Reports, 40, 1135-41.

Schmidt, H.O., Fonda, C.P., 1954. Rorschach scores in the manic state. Journal of Psychology 38, $427-437$.

Shenton, M.E., Solovay, M.R., Holzman, P.S., 1987. Comparative studies of thought disorders II: schizoaffective disorder. Archives of General Psychiatry 44, 21-30.

Singer, H.K., Brabender, V., 1993. The use of the Rorshach to differentiate unipolar and bipolar disorders. Journal of Personality Assessment 60, 333-345.

Solovay, M.R., Shenton, M.E., Holzman, P.S., 1987. Comparative studies of thought disorders I: mania and schizophrenia. Archives of general Pshychiatry 44, 13-20.

Wagner, E.E., Heise, M.R., 1981. Rorschach and hand test data comparing bipolar patients in manic and depressive phases. Journal of Personality Assessment 45, 240-249.

Wagner, E.E., Rinn, R.C., 1994. The extrapolated area response: a pathognomic sign of mania on the Rorschach? Perceptual and Motor Skills 78, 1025-1026.

Weiner, I.B., 2003. Principles of Rorschach Interpretation. Lawrence Erlbaum Associates, Mahwah.

Worland, J., 1979. Rorschach developmental level in the offspring of patients with schizophrenia and manic-depressive illness. Journal of Personality Assessment 43, 591-594. 